\title{
Global fits to neutrino oscillations: status and prospects
}

\section{Antonio Marrone*}

Dipartimento di Fisica and Sezione INFN di Bari, Via Amendola 173, 70126 Bari, Italy

E-mail: antonio.marronedba.infn.it

\section{Eligio Lisi}

Istituto Nazionale di Fisica Nucleare, Sezione di Bari, Via Orabona 4, 70126 Bari, Italy

\section{Francesco Capozzi}

Dipartimento di Fisica and Sezione INFN di Bari, Via Amendola 173, 70126 Bari, Italy

\section{Daniele Montanino}

Dipartimento di Matematica e Fisica and Sezione INFN di Lecce, Via Arnesano, 73100 Lecce, Italy

\section{Antonio Palazzo}

Dipartimento di Fisica and Sezione INFN di Bari, Via Amendola 173, 70126 Bari, Italy

In this work we review our present knowledge of the neutrino oscillation parameters. In a threeneutrino framework, neutrino oscillations depend on six parameters, two squared mass differences $\left(\Delta m^{2}, \delta m^{2}\right)$, three mixing angles $\left(\theta_{12}, \theta_{13}, \theta_{23}\right)$ and one phase $\delta$. Five out of these six parameters have been measured by a number of experiments and only the CP-violating phase $\delta$ remains unknown. Moreover, the octant of the mixing angle $\theta_{23}$ and the neutrino mass hierarchy are still undetermined. We update our previous 2014 analysis, by adding to the global fit the recent results of the antineutrino running of $\mathrm{T} 2 \mathrm{~K}$ and the first results of the NOvA experiment.

The European Physical Society Conference on High Energy Physics 22-29 July 2015

Vienna, Austria

\footnotetext{
*Speaker.
} 


\section{Introduction}

The three-neutrino mass-mixing framework is nowadays well established and can explain almost all $v$ oscillation data [1]. In this framework, the flavor eigenstates $v_{\alpha}(\alpha=e, \mu, \tau)$ are a superposition of the mass eigestates $v_{i}(i=1,2,3)$ through the three mixing angles $\left(\theta_{12}, \theta_{13}, \theta_{23}\right)$ and a $\mathrm{CP}$-violating phase $\delta$. However, neutrino oscillations depend on the neutrino masses $m_{i}$ through two quantities that in our convention are chosen as $\delta m^{2}=m_{2}^{2}-m_{1}^{2}>0$ and $\Delta m^{2}=$ $m_{3}^{2}-\left(m_{1}^{2}+m_{2}^{2}\right) / 2$. While the sign of $\delta m^{2}$ is always positive, the sign of $\Delta m^{2}$ distinguishes two possible ordering of the mass eigenstates, the normal hierarchy (NH), when $\Delta m^{2}>0$, and the inverted hierarchy (IH) in the opposite case [2]. Five out of the six above oscillation parameters have been measured by a number of experiments [3]. In particular, the two "solar" parameters $\left(\delta m^{2}, \theta_{12}\right)$ have been measured by solar experiments in combination with KamLAND, $\theta_{13}$ by short-baseline experiments (SBL) and the "atmospheric" parameters $\left(\Delta m^{2}, \theta_{23}\right)$ by atmospheric and long-baseline (LBL) experiments. However, it is still unknown if the mixing angle $\theta_{23}$ is close to maximal or not, and in this second case, which is its octant. Secondly, the precise value of the phase $\delta$ is also still unknown, even though very recent data begin to constrain its allowed range at 2-3 $\sigma$ level. Finally, present data, even in the global analysis, are only poorly sensitive to the hierarchy discrimination. Global analyses are a very useful tool to verify the consistency of all available oscillation data and can also, as in the past for the $\theta_{13}$ mixing angle, give some hints about parameters that are not well constrained by a single class of experiments. In this work we report about our present knowledge about the oscillation parameters, in view of the latest available experimental results and discuss

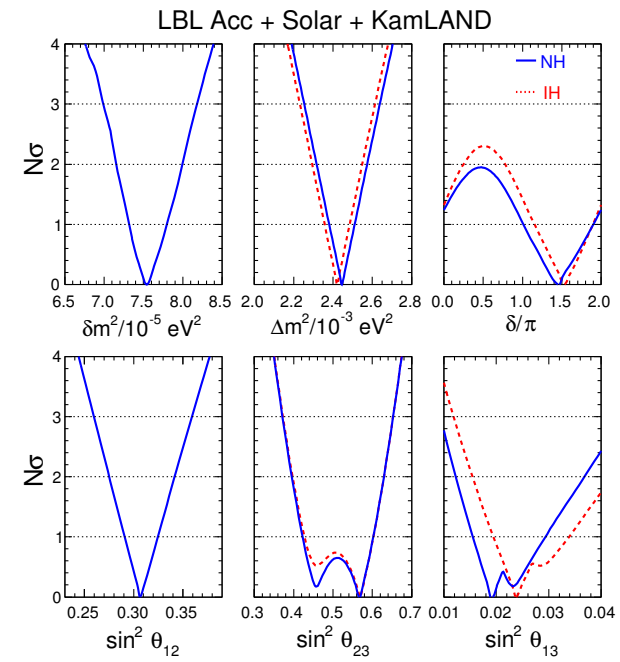

Figure 1: Combined analysis of LBL, Solar, and KamLAND data. Solid blu lines refer to $\mathrm{NH}$, red dashed lines to $\mathrm{IH}$.

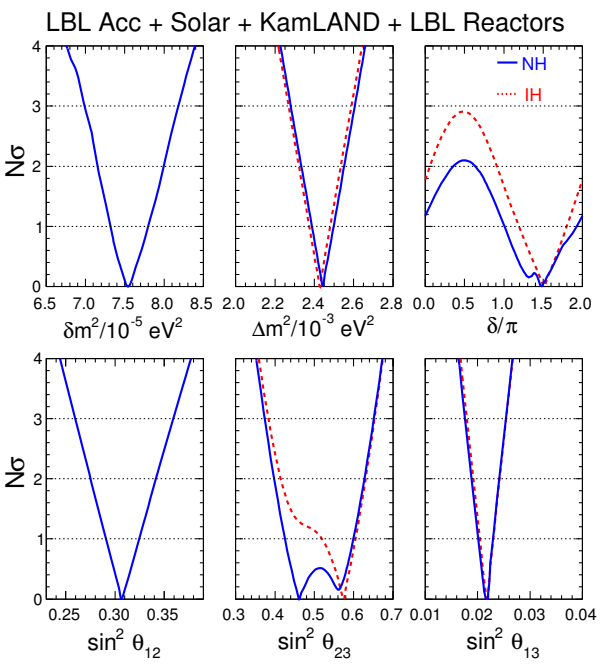

Figure 2: As in Figure 1, but adding SBL data. 
$\mathrm{LBL}$ Acc + Solar + KamLAND + LBL Reactors + SK Atm

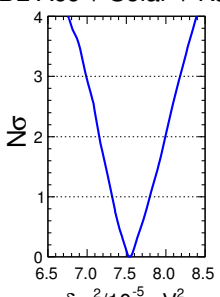
$\delta \mathrm{m}^{2} / 10^{-5} \mathrm{eV}^{2}$
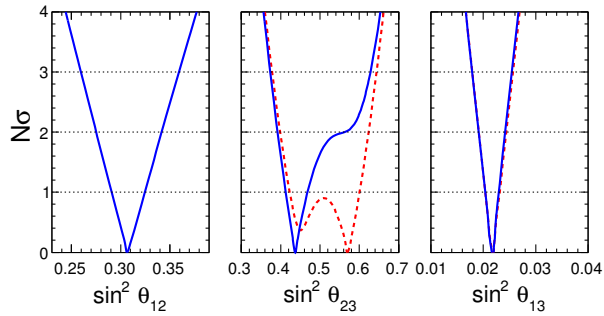

Figure 3: Global fit of all data (NOvA LID).
LBL Acc + Solar + KamLAND + LBL Reactors + SK Atm
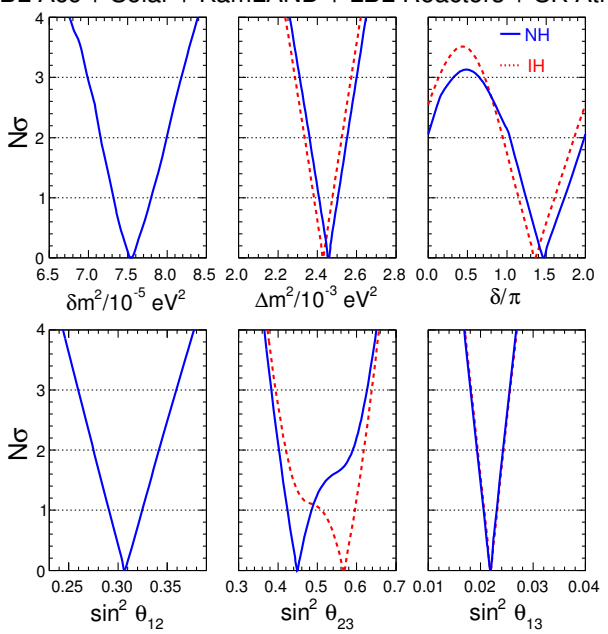

Figure 4: Global fit of all data (NOvA LEM).

future prospects about the unknown ones. We will upgrade our previous analysis by including in the global fit the latest T2K [4] and NOvA [5] results.

\section{Methodology of the global analysis}

We divide the data samples in different subsets and combine them in such a way to fully exploit parameter correlations, to understand what is effectively happening in the global fit. In particular we divide our data in five subsets: LBL, KamLAND, solar, SBL, and atmospheric neutrino data. We include in our analysis of LBL data the experimental results of MINOS, T2K, NOvA and K2K and in the SBL analysis Daya Bay, RENO and Double Chooz data.

Firstly, we combine solar and KamLAND data with LBL results. The solar parameters $\left(\delta m^{2}, \theta_{12}\right)$ are well constrained by solar + KamLAND analysis, and can be essentially fixed at their best-fit values in the subsequent LBL analysis. The oscillation probability for LBL accelerator experiments is mostly dependent on the atmospheric parameters $\left(\Delta m^{2}, \theta_{23}\right)$ in the $v_{\mu} \rightarrow v_{\mu}$ disappearance channel, it depends also on $\theta_{13}$ in the $v_{\mu} \rightarrow v_{e}$ appearance channel, while it is only subdominantly dependent on the solar parameters and $\delta$. This is the reason why we initially combine LBL, solar and KamLAND data. In our analysis, the best-fit value for $\theta_{13}$ from solar+KamLAND data $\left(\sin ^{2} \theta_{13} \sim 0.02\right)$ is a bit larger than the one measured at SBL experiments. This weak preference for a non-zero $\theta_{13}$ is improved when adding LBL data, providing a significant measurement of $\theta_{13}$. However, the obtained best-fit value of $\theta_{13}$ is sensitive to the precise value of $\delta$ and $\theta_{23}$. Secondly, we add to our analysis the SBL results that provide a very precise value of $\theta_{13}$, independent 

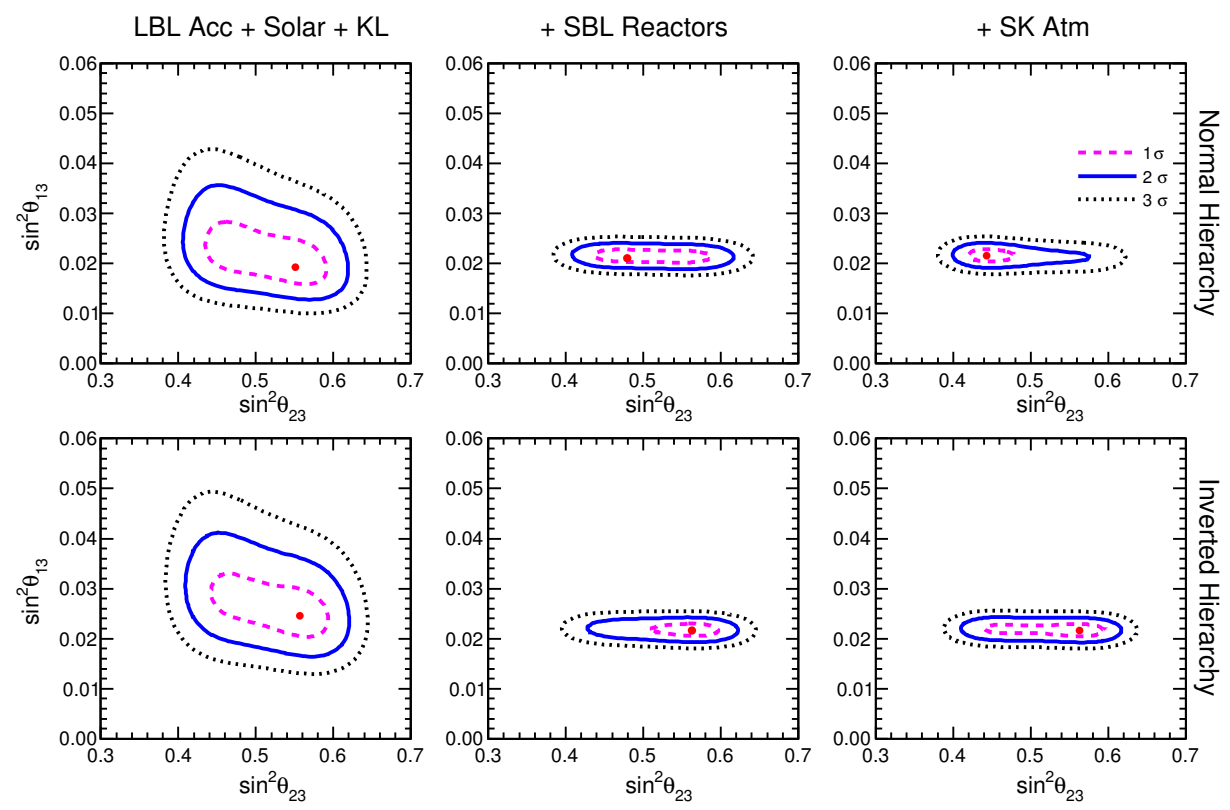

Figure 5: Results of the analysis in the plane $\left(\sin ^{2} \theta_{23}, \sin ^{2} \theta_{13}\right)$.

on $\delta$ and $\theta_{23}$, and finally we add the SuperKamiokande results on atmospheric neutrinos. Atmospheric neutrino data are sensitive to the $\theta_{23}$ octant and also, although very weakly, to the phase $\delta$. When reporting the results of our analysis we define $N$-sigma allowed regions by the relation $N \sigma=\sqrt{\chi^{2}-\chi_{\min }^{2}}$, while the relative preference for the hierarchy is quantified by the difference $\Delta \chi_{I-N}^{2}=\chi_{\min }^{2}(I H)-\chi_{\min }^{2}(N H)$.

\section{Single parameter analysis}

In this section the results on each of the six oscillation parameter is shown, for the three cases discussed in the introduction. Blu solid curves refer to $\mathrm{NH}$, while red dashed ones are for $\mathrm{IH}$. In Figure 1, LBL, Solar and KamLAND data are combined. Since Solar+KamLAND data are practically insensitive to the hierarchy, the curves for $\mathrm{NH}$ and IH basically coincide for $\delta m^{2}$ and $\theta_{12}$. Bounds on all the parameters are obtained with the exception of $\delta$. However, the bounds from LBL data on $\theta_{13}$ (dominated by T2K $v_{\mu} \rightarrow v_{e}$ channel and now corroborated by T2K disappearance and NOvA recent results) induce an intriguing preference for $\delta \sim 1.5 \pi$. It is worth noticing that $\mathrm{T} 2 \mathrm{~K}$ and NOvA data require require the maximization of the appearance probability and hence $\sin \delta \sim-1$, dominating in the fit over the MINOS preference for $\sin \delta>0$. With regard to the octant of $\theta_{23}$, slightly non-maximal $\theta_{23}$ mixing is preferred by MINOS disappearance data. In Figure 2, SBL data are added to the fit. Consequently, the uncertainty on $\theta_{13}$ is strongly reduced, the octant of $\theta_{23}$ is swapped for $\mathrm{NH}$ and the preference for negative $\sin \delta<0$ slightly increased.

Finally, by adding the atmospheric data, the preference for $\sin \delta>0$ is reinforced, even though 

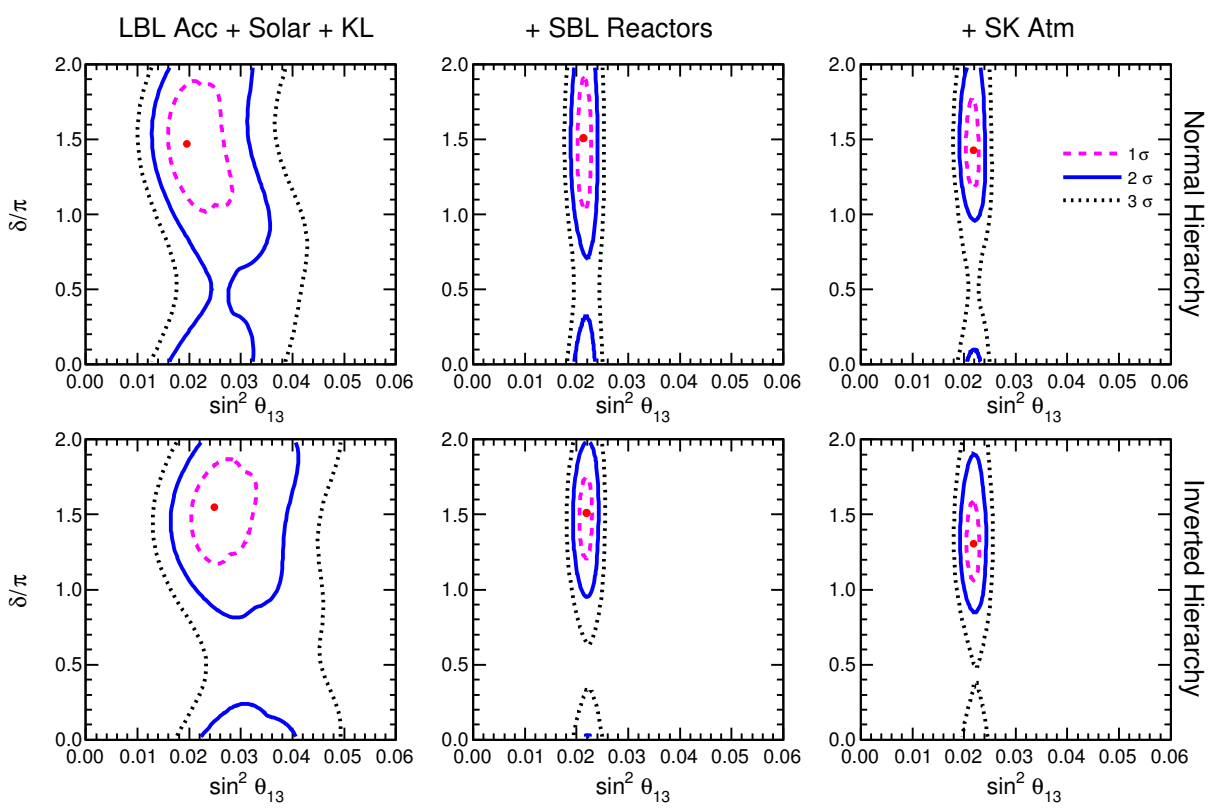

Figure 6: Results of the analysis in the plane $\left(\sin ^{2} \theta_{13}, \delta\right)$.

with a slight low best-fit value and a more pronounced preference for non-maximal $\theta_{23}$ is found. The CP-conserving cases $\delta=0, \pi$ are still allowed from the global fit at about $2 \sigma$ level in both hierarchies. The latest T2K and NOvA data give bounds on $\delta$ consistent with the previous analysis, disfavouring $\delta \sim 0.5 \pi$ at about 2.8 $\sigma$. However, the NOvA collaboration presented two independent data analysis, that they call LID (the one used in Fig. 1-3) and LEM. In Figure 4 the global fit results with the NOvA LEM analysis are shown. In this case the preference for $\sin \delta \sim-1$ is more pronounced and $\delta \sim 0.5 \pi$ is disfaoured at more than $3 \sigma$. At present, the global analysis does not give statistically significant information on the hierarchy: the NH is preferred, with the $\Delta \chi_{I-N}^{2}$ difference of about 0.3 (with NOvA LID data) or about 2.2 (with NOvA LEM data).

\section{Two parameter covariances}

In this section some of the correlation between the oscillation parameters are shown and discussed. Figure 5 shows the allowed regions in the $\left(\theta_{23}, \theta_{13}\right)$ plane. The three columns refer to increasingly reach data set, for NH (top) and IH (bottom). In the first column it can be seen that there is a weak anticorrelation between the two mixing angles, coming from the LBL appearance data, because the oscillation probability contains a term proportional to the product $\sin ^{2} \theta_{13} \sin ^{2} \theta_{23}$. The strong appearance signals in $\mathrm{T} 2 \mathrm{~K}$, both in the neutrino and antineutrino channels, and in NOvA require relatively higher $\theta_{13}$ values, while Solar+KamLAND prefer $\sin ^{2} \theta_{13} \sim 0.02$. This is the reason why the best fit for $\theta_{23}$ is in the second octant, for relatively low $\sin ^{2} \theta_{13}$, for both hierarchies. In the second column, when SBL results are included, the $\sin ^{2} \theta_{13}$ best-fit point moves to 0.023 , and for $\mathrm{NH}$ this causes the swap of the $\theta_{23}$ octant. The inclusion of the atmospheric data (third 

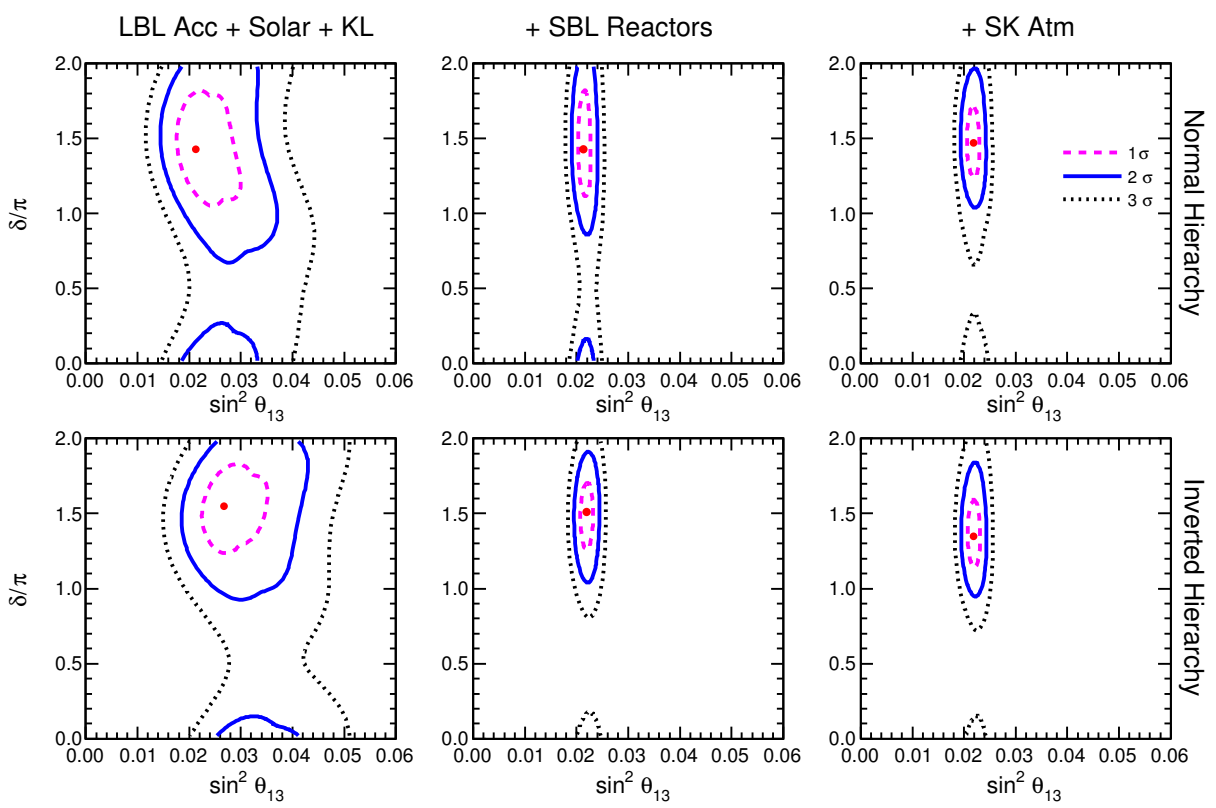

Figure 7: Results of the analysis in the plane $\left(\sin ^{2} \theta_{13}, \delta\right)$ with LEM NOvA data.

column) does not change the preferred $\theta_{23}$ octant, the first one being preferred for $\mathrm{NH}$, the second one for IH.

Figure 6 shows the allowed regions in the $\left(\theta_{13}, \delta\right)$ plane. In the first panel it is evident how the preference for $\delta \sim 1.5 \pi$ originates form a compromise between the relatively low value of $\theta_{13}$ from Solar+KamLAND data and the higher value required by the appearance signals of T2K and NOvA. With respect to our previous global analysis [3], the allowed regions are reduced, and there is a range around $\delta \sim 0.5 \pi$ excluded at $2 \sigma$ for IH. When SBL results are added the preference for $\delta \sim 1.5 \pi$ remains and the covariance between the two parameters is strongly reduced. The inclusion of the atmospheric SK data does not significatively alter this trend but slightly moves the best-fit point of $\delta$ to lower values. Figure 7 shows the same correlations of Figure 6, but with the NOvA LEM data. In this case the size of the $\delta$ allowed regions is reduced in both hierarchies.

\section{Conclusions}

In this work we presented an update of the global analysis of the available oscillation neutrino data, including the recent $\mathrm{T} 2 \mathrm{~K}$ antineutrino results and the first NOvA results. We have updated the $N \sigma$ bounds on the oscillation parameters $\left(\Delta m^{2}, \delta m^{2}, \theta_{12}, \theta_{13}, \theta_{23}\right)$ and discussed the current information about the phase $\delta$ and the octant of $\theta_{23}$. Concerning the phase $\delta$, we find an intriguing preference for $\delta \sim 1.5 \pi$ and in general for $\sin \delta<0$. The value $\delta=\pi / 2$ is now disfavoured at about $3 \sigma$. The determination of the octant of $\theta_{23}$ is currently unstable, depending on the hierarchy and on the different data sets included in the fit. There is currently no clear indication in favour of one hierarchy, even though a weak preference for NH emerges in the case of the NOVA LEM 
analysis. Concerning the phase $\delta$ and the octant of $\theta_{23}$, the increasing statistics at the LBL experiments, will certainly improve the bounds on these two parameters in the next few years, but with a significativity depending on the possible degeneracies between the oscillation parameters and the true hierarchy. For the determination of the hierarchy there are very interesting experimental projects $[6,7,8,9]$, able to achieve this goal on a time scale of five to ten years. However, these projects are very challenging and their success will depend on an accurate evaluation of all systematics [10] at a percent level or better.

\section{References}

[1] K.A. Olive et al. (Particle Data Group), Chin. Phys. C 38, 090001 (2014). See the review therein: "Neutrino mass, mixing and oscillations," by K. Nakamura and S.T. Petcov.

[2] G.L. Fogli, E. Lisi, A. Marrone, A. Palazzo, Prog.Part.Nucl.Phys. 57 (2006) 742-795.

[3] F. Capozzi, G. L. Fogli, E. Lisi, A. Marrone, D. Montanino and A. Palazzo, Phys. Rev. D 89, 093018 (2014) [arXiv:1312.2878 [hep-ph]].

[4] M. Ravonel, talk at this Conference.

[5] B. Rebel, talk at TAUP 2015 Conference, 6-12 September, Torino, Italy.

[6] M. Wurm, talk at TAUP 2015 Conference, 6-12 September, Torino, Italy.

[7] Seon-Hee Seo, talk at TAUP 2015 Conference, 6-12 September, Torino, Italy.

[8] T. Ehrhardt, talk at this Conference.

[9] T. Pradier, talk at this Conference.

[10] F. Capozzi, E. Lisi, A. Marrone, arXiv:1508.01392 [hep-ph],

F. Capozzi, E. Lisi, A. Marrone, Phys. Rev. D 91 (2015) 073011, [arXiv:1503.01999 [hep-ph]],

F. Capozzi, E. Lisi, A. Marrone, Phys. Rev. D 89 (2014) 1, 013001. 